\title{
The WHILE Hierarchy of Program Schemes Is Infinite
}

\author{
Can Adam Albayrak and Thomas Noll \\ RWTH Aachen \\ Ahornstr. 55, 52056 Aachen, Germany \\ e-mail: albayrak@informatik.ruth-aachen.de and \\ nollQinformatik. rwth-aachen. de \\ fax: +492418888217
}

\begin{abstract}
We exhibit a sequence $S_{n}(n \geq 0)$ of WHILE program schemes, i. e., WHILE programs without interpretation, with the property that the WHILE nesting depth of $S_{n}$ is $n$, and prove that any WHILE program scheme which is scheme equivalent to $S_{n}$, i. e., equivalent for all interpretations over arbitrary domains, has WHILE nesting depth at least $n$. This shows that the wHILE nesting depth imposes a strict hierarchy (the wHILE hierarchy) when programs are compared with respect to scheme equivalence and contrasts with Kleene's classical result that every program is equivalent to a program of wHILE nesting depth 1 (when interpreted over a fixed domain with arithmetic on non-negative integers). Our proof is based on results from formal language theory; in particular, we make use of the notion of star height of regular languages.
\end{abstract}

\section{Introduction}

When comparing programming languages, one often has a vague impression of one language being more powerful than another. However, a basic result of the theory of computability is that even simple models of computation like Turing machines, WHILE programs (with arithmetic), and partial recursive functions are universal in the following sense: They describe exactly the class of computable functions, according to Church's thesis. The proof uses encodings of functions by non-negative integers with the help of zero and successor function. Thus, if the programming language under consideration supports arithmetic on nonnegative integers, then it is capable of simulating any effective control structure.

A compiler implementing a programming language could in principle adopt this method. In general, such languages do not only specify computations over non-negative integers but they handle data types like floating-point numbers, character strings, and trees as well. Additionally, modern programming languages allow recursion as means for the description of algorithms. In principle, these extended capabilities could be implemented (1) by embedding them in the setting of non-negative integers using appropriate encodings, (2) by simulating 
their behavior as a computable function, and (3) by translating the result back into the original context. However, it is clear that this approach is of purely theoretical interest; there is no hope for achieving good efficiency in this way. Instead these concepts are implemented directly: for example recursion is usually translated into iterative algorithms using a run-time stack.

Thus a comparison of the computational power of programming languages requires the distinction between the control structures of a program and other aspects like the semantic domains involved in the computations. Therefore we use the approach to decompose a program into a program scheme and an interpretation (which comprises the semantic domain). We study only the scheme part as an abstraction of the family of all programs represented by this scheme. In this generalized approach, two schemes are considered to be equivalent iff the concrete programs obtained by addition of an interpretation are equivalent for all interpretations. It is well-known that the schematic concept of "recursion" is more powerful than that of "iteration" [12], that "recursion" equals "iteration + stack" [2], and that "iteration" equals "while + Boolean variables" [1].

Unfortunately the question of scheme equivalence is undecidable in the general case [11]. The reason is not, as one might expect, the "large number" of interpretations which one has to apply for deciding scheme equivalence - it suffices to consider free interpretations (or Herbrand interpretations) only. Instead, the undecidability is caused by the structure of the state space of the program, more precisely the state space has too many components. If we abstract from the state space we obtain simple or monadic schemes for which the question of scheme equivalence becomes decidable in most cases.

In this paper we consider the class of Dijkstra schemes which are inductively built up from atomic statements by means of sequential composition, branching instructions, and WHILE loops. A characterization of scheme equivalence via regular languages is exploited: the star height of the regular language associated with a Dijkstra scheme yields a lower bound for the WHILE nesting depth required. We exhibit a sequence $S_{n}(n \geq 0)$ of Dijkstra schemes with the property that the WHILE nesting depth of $S_{n}$ is $n$, and prove (via the correspondence to regular languages) that any Dijkstra scheme which is equivalent to $S_{n}$ has WHILE nesting depth at least $n$. This shows that the wHILE nesting depth of Dijkstra schemes imposes a strict hierarchy with respect to the computational power of the corresponding class of programs - the wHILE hierarchy. It contrasts with Kleene's classical result [10] that every program is equivalent to a program of WHILE nesting depth 1 (beside some fixed number of other loops) when interpreted over a fixed domain with arithmetic on non-negative integers.

\section{Dijkstra schemes}

Here we introduce the class of Dijkstra schemes. The only construction elements for Dijkstra schemes are sequential composition, branching, and conditional it- 
eration. Thus, Dijkstra schemes can be regarded as WHILE programs without interpretations.

Let $\Omega, \Pi$ be non-empty, finite, and disjoint sets of unary function symbols and unary predicate symbols. $(\Omega, \Pi)$ is called a signature. The set $\operatorname{BExp}(\Pi)$ of Boolean expressions over $\Pi$ is the smallest set which contains $\Pi$ and which is closed under the Boolean operations (i. e. $\wedge, \vee$ and $\neg$ ). The class $\operatorname{Dij}(\Omega, \Pi)$ of Dijkstra schemes over $\Omega$ and $\Pi$ is the smallest set which, for every $S, S_{1}, S_{2} \in$ $\operatorname{Dij}(\Omega, \Pi)$ and $b \in \operatorname{BExp}(\Pi)$, satisfies the following conditions:

$-\Omega \subseteq \operatorname{Dij}(\Omega, \Pi)$

- $\left(S_{1} ; S_{2}\right) \in \operatorname{Dij}(\Omega, \Pi)$

- if $b$ then $S_{1}$ else $S_{2}$ fi $\in \operatorname{Dij}(\Omega, \Pi)$

- while $b$ do $S$ done $\in \operatorname{Dij}(\Omega, \Pi)$.

We allow to omit braces.

Example 1. Let $\Omega:=\{f, g, h\}$ and $\Pi:=\{p\}$. Then

$$
\begin{aligned}
& f \text {; } \\
& \text { while } p \text { do } \\
& (g ; h) \\
& \text { done }
\end{aligned}
$$

is a Dijkstra scheme.

A $(\Omega, \Pi)$-interpretation, or interpretation for short, is a pair $\mathcal{A}:=\langle A ; \alpha\rangle$ where $A$ is a non-empty set, the domain of the interpretation, and $\alpha$ is a mapping which assigns a predicate $\alpha(p): A \rightarrow\{0,1\}$ to every symbol $p \in \Pi$ and a total function $\alpha(f): A \rightarrow A$ to every symbol $f \in \Omega$. Instead of $\alpha(f)$ we write $f_{\mathcal{A}}$. The class of all $(\Omega, \Pi)$-interpretations is denoted by $\operatorname{Int}(\Omega, \Pi)$.

A pair $(S, \mathcal{A})$ consisting of a Dijkstra scheme $S \in \operatorname{Dij}(\Omega, \Pi)$ and an interpretation $\mathcal{A} \in \operatorname{Int}(\Omega, \Pi)$ is called a Dijkstra program. The semantics of $(S, \mathcal{A})$ is the (partial) mapping $\llbracket S \rrbracket_{\mathcal{A}}: A \rightarrow A$, given as follows:

$$
\llbracket S \rrbracket_{\mathcal{A}}(a):= \begin{cases}f_{\mathcal{A}}(a) & , \text { if } S \in \Omega \\ \llbracket S_{2} \rrbracket_{\mathcal{A}}\left(\llbracket S_{1} \rrbracket_{\mathcal{A}}(a)\right) & , \text { if } S=\left(S_{1} ; S_{2}\right) \\ \llbracket S_{1} \rrbracket_{\mathcal{A}}(a) & , \text { if } S=\text { if } b \text { then } S_{1} \text { else } S_{2} \text { fi and } \llbracket b \rrbracket_{\mathcal{A}}(a)=1 \\ \llbracket S_{2} \rrbracket_{A}(a) & , \text { if } S=\text { if } b \text { then } S_{1} \text { else } S_{2} \text { fi and } \llbracket b \rrbracket_{\mathcal{A}}(a)=0 \\ \llbracket S^{\prime} \rrbracket_{\mathcal{A}}^{k}(a) & , \text { if } S=\text { while } b \text { do } S^{\prime} \text { done } \\ & \text { and the WHILE condition holds } \\ \text { undefined } & \text { else }\end{cases}
$$

where $\llbracket b \rrbracket_{\mathcal{A}}(a)$ is the truth value of $b$ on input $a$ which is induced by the interpretation $\mathcal{A}$ and where the WHILE condition depending on $b, S$ and $a$ is given by

$$
\forall i \in\{0, \ldots, k-1\}: \llbracket b \rrbracket_{\mathcal{A}}\left(\llbracket S^{\prime} \rrbracket_{\mathcal{A}}^{i}(a)\right)=1 \text { and } \llbracket b \rrbracket_{\mathcal{A}}\left(\llbracket S^{\prime} \rrbracket_{\mathcal{A}}^{k}(a)\right)=0 \text {. }
$$


As usual $\llbracket b \rrbracket_{\mathcal{A}}\left(\llbracket S^{\prime} \rrbracket_{\mathcal{A}}^{i}(a)\right)=1$ means that the $i$ th iteration $\llbracket S^{\prime} \rrbracket_{\mathcal{A}}^{i}$ of the mapping $\llbracket S^{\prime} \rrbracket_{\mathcal{A}}$ applied to $a$ is defined and that the results satisfies condition $b$.

Example 2. Let $S$ the Dijkstra scheme in Example 1 and $\mathcal{A}:=\left\langle\mathbf{N}^{2} ; \alpha\right\rangle(\mathbf{N}$ is the set of all non-negative integers) with $f_{\mathcal{A}}(m, n):=(m, 1), g_{\mathcal{A}}(m, n):=(m, m \cdot n)$, $h_{\mathcal{A}}(m, n):=(\max \{0, m-1\}, n)$ and $p_{\mathcal{A}}(m, n)=1 \Longleftrightarrow m \neq 0$. Then $\llbracket S \rrbracket_{\mathcal{A}}$ computes the factorial function, more precisely $\llbracket S \rrbracket_{\mathcal{A}}(m, n)=(0, m !)$.

As mentioned in the introduction we define:

Two Dijkstra schemes $S_{1}, S_{2} \in \operatorname{Dij}(\Omega, \Pi)$ are (strongly) equivalent iff the equation

$$
\llbracket S_{1} \rrbracket_{\mathcal{A}}=\llbracket S_{2} \rrbracket_{\mathcal{A}}
$$

is valid for all interpretations $\mathcal{A} \in \operatorname{Int}(\Omega, \Pi)$. We write $S_{1} \sim S_{2}$ iff $S_{1}$ and $S_{2}$ are equivalent.

Hence scheme equivalence comprises program equivalence which expresses that, under a fixed interpretation, both programs compute the same function.

\section{Characterization of Dijkstra scheme equivalence}

Now we give a characterization of scheme equivalence in terms of formal languages. The language $L_{S}$ which we associate with a given Dijkstra scheme $S$ is a regular language capturing the full computation potential of $S$. To simulate the behaviour of $S$ under arbitrary interpretations, we especially record the decisions which have been taken in the Boolean conditions. The languages we define use Boolean vectors for this protocol; a word of this language consists of function symbols and Boolean vectors in alternation. The central point is the representation of scheme composition by a conditional product [9] of the corresponding languages. It allows two computations to be concatenated only if their adjacent Boolean vectors coincide.

For a set $\Omega$ of unary function symbols and a set $\Pi=\left\{p_{1}, \ldots, p_{n}\right\}$ of predicate symbols with $n$ elements let

$$
\mathbf{B}:=\{0,1\}^{n}
$$

be the set of all Boolean vectors of length $n$. We associate with each Boolean expression $b \in \mathbf{B E x p}(\Pi)$ a set of Boolean vectors $L_{b} \subseteq \mathbf{B}$ by induction: for $p_{i} \in \Pi$ let

$$
L_{p_{i}}:=\left\{\left(x_{1}, \ldots, x_{i-1}, 1, x_{i+1}, \ldots, x_{n}\right) \mid x_{1}, \ldots, x_{i-1}, x_{i+1}, \ldots, x_{n} \in\{0,1\}\right\},
$$

and $L_{b_{1} \wedge b_{2}}:=L_{b_{1}} \cap L_{b_{2}}, L_{b_{1} \vee b_{2}}:=L_{b_{1}} \cup L_{b_{2}}$, and $L_{\neg b}:=\mathbf{B} \backslash L_{b}$. Now we are ready to specify the Dijkstra scheme language $L_{S}$ of an arbitrary scheme 
$S \in \operatorname{Dij}(\Omega, \Pi)$. It is given by the following inductive definition:

$$
\begin{aligned}
& L_{f}:=\mathbf{B} \cdot\{f\} \cdot \mathbf{B} \\
& L_{\left(S_{1} ; S_{2}\right)}:=L_{S_{1}} \circ L_{S_{2}} \\
& L_{\text {if } b \text { then } S_{1} \text { else } S_{2} \mathbf{f}}:=\left(L_{b} \circ L_{S_{1}}\right) \cup\left(\left(\mathbf{B} \backslash L_{b}\right) \circ L_{S_{2}}\right) \\
& L_{\text {while } b \text { do } S \text { done }}:=\left(\bigcup_{i \in \mathbf{N}}\left(L_{b} \circ L_{S}\right)^{i}\right) \circ\left(\mathbf{B} \backslash L_{b}\right)
\end{aligned}
$$

where $L_{1} \circ L_{2}$ is the conditional product defined by

$$
L_{1} \circ L_{2}:=\left\{w \beta v \mid \beta \in \mathbf{B}, w \in(\mathbf{B} \Omega)^{*}, v \in(\Omega \mathbf{B})^{*}, w \beta \in L_{1} \text { and } \beta v \in L_{2}\right\}
$$

and $L^{0}:=\mathbf{B}$ and $L^{i+1}:=L^{i} \circ L$ for every $i \in \mathbf{N}$. The class of all Dijkstra scheme languages over $\Omega$ and $\Pi$ is denoted by $\mathcal{L}_{\mathbf{D i j}}(\Omega, \Pi)$.

Example 3. Let $S$ be the Dijkstra scheme in Example 1, and let $B:=(0+1)$. Then $L_{S}$ is the language denoted by the regular expression

$$
B f(1 g B h)^{*} 0 \text {. }
$$

Proposition 4. (Characterization of Dijkstra scheme equivalence) For any two Dijkstra schemes $S_{1}, S_{2} \in \operatorname{Dij}(\Omega, \Pi)$ the following condition holds:

$$
S_{1} \sim S_{2} \Longleftrightarrow L_{S_{1}}=L_{S_{2}} .
$$

Proof. It is well-known that every Dijkstra scheme is translatable into an equivalent Ianov scheme, which can be considered as an uninterpreted (monadic) flowchart (see [7], [6], [8], and [13] for further details). For this class of schemes, I. I. Ianov gave a language-theoretic description of equivalence by assigning to every scheme a deterministic finite automaton whose recognized language characterizes the scheme equivalence. The combination of both techniques yields our proof: by induction on the syntactic structure of $S \in \operatorname{Dij}(\Omega, \Pi)$ it is possible to show that the language associated with the equivalent Ianov scheme $\operatorname{trans}(S)$ and the language $L_{S}$ assigned to $S$ coincide.

Example 5. Let $S$ once again be the Dijkstra scheme in Example 1 and $S^{\prime}$ be the Dijkstra scheme

$$
\begin{aligned}
& f ; \\
& \text { if }(\neg p) \text { then } \\
& \text { while } p \text { do }(g ; h) \text { done } \\
& \text { else } \\
& \quad(g ; h) ; \\
& \quad \text { while } p \text { do }(g ; h) \text { done } \\
& \text { fi }
\end{aligned}
$$

Let $B:=(0+1)$. Then the Dijkstra scheme language $L_{S^{\prime}}$ is the language denoted by the regular expression

$$
(B f 0)+\left(B f 1 g B h(1 g B h)^{*} 0\right) .
$$

Since it is the same language as the Dijkstra scheme language for $S$ we can deduce by Proposition 4 that $S$ and $S^{\prime}$ are equivalent. 


\section{The star height of regular languages}

In order to prove the main theorem we use the concept of star height of regular languages. After presenting some known facts concerning the star height, we show that there exists an infinite family of regular languages $\left(L_{n}\right)_{n \in \mathbf{N}}$ such that every language $L_{n}$ of this family has star height $n$. This knowledge will be exploited in the next section.

We use $\emptyset$ to denote the empty language, $\varepsilon$ to denote the language which consists of the empty word and $L(\alpha)$ for the language denoted by the regular expression $\alpha$. The set of all regular expressions over a finite alphabet $\Sigma$ is denoted by $\mathbf{R E}(\Sigma)$.

The star height of a regular expression is the maximal number of nested stars which appear in this expression, and the star height of a regular language is the minimal star height of all regular expressions denoting this language, more formally one defines for a finite alphabet $\Sigma$

$$
\begin{aligned}
& -\operatorname{sh}(\emptyset)=\operatorname{sh}(\varepsilon)=\operatorname{sh}(a)=0 \text { for all symbols } a \in \Sigma \\
& -\operatorname{sh}(\alpha \beta)=\operatorname{sh}(\alpha+\beta)=\max \{\operatorname{sh}(\alpha), \operatorname{sh}(\beta)\} \text { for } \alpha, \beta \in \mathbf{R E}(\Sigma) \\
& -\operatorname{sh}\left(\alpha^{*}\right)=\operatorname{sh}(\alpha)+1 \text { for } \alpha \in \mathbf{R E}(\Sigma),
\end{aligned}
$$

and for a regular language $L \subseteq \Sigma^{*}$

$$
\operatorname{sh}(L):=\min \{\operatorname{sh}(\alpha) \mid \alpha \in \mathbf{R E}(\Sigma) \text { and } L(\alpha)=L\}
$$

is called the star height of $L$.

Example 6. (Star height [3]) Let $\Sigma:=\{a, b\}$. The regular expression

$$
\left(b a^{*} b\right)^{*}
$$

has star height 2 but the language $L\left(\left(b a^{*} b\right)^{*}\right)$ denoted by this regular expression has at most star height 1 , because

$$
L\left(\varepsilon+b(a+b b)^{*} b\right)=L\left(\left(b a^{*} b\right)^{*}\right)
$$

and $\operatorname{sh}\left(\varepsilon+b(a+b b)^{*} b\right)=1$. Furthermore it is easy to show that a regular language is finite iff it has zero star height. So we get $\operatorname{sh}\left(L\left(\left(b a^{*} b\right)^{*}\right)\right)=1$.

In 1963 L. C. Eggan has raised up the question whether there are languages of arbitrary star height over a two letter alphabet [5]. F. Dejean and M. P. Schützenberger gave a positive answer to this question by showing that for every $n \in \mathbb{N} \backslash\{0\}$ the language $L_{n}$ over the alphabet $\{a, b\}$ which is recognized by the deterministic finite automaton $\mathcal{A}_{n}$ 


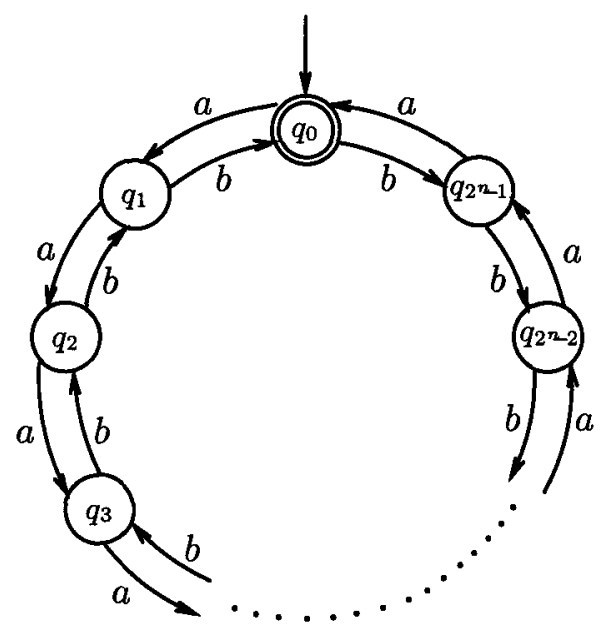

with $2^{n}$ states has star height $n$. We pick up the technique which has been used in [4] for showing that in a special subclass of regular languages, the class $\mathcal{L}_{\text {Dij }}(\Omega, \Pi)$ of all Dijkstra scheme languages, there also exist languages of arbitrary star height.

The following well-known lemma, which we need in the next section, is easy to prove.

Lemma 7. (Star height of homomorphic images) Let $\Sigma$ be a finite alphabet and $h: \Sigma^{*} \rightarrow \Sigma^{*}$ be a homomorphism on $\Sigma^{*}$. Then for every regular language $L \subseteq \Sigma^{*}$ :

$$
\operatorname{sh}(h(L)) \leq \operatorname{sh}(L) .
$$

The next lemma presents the regular language family by which we are going to establish the connection to Dijkstra schemes.

Lemma 8. (Star height of a certain family of regular languages) Let $\left(\alpha_{n}\right)_{n \in \mathrm{N}}$ be a family of regular expressions over the alphabet $\Sigma:=\{f, g\}$, defined inductively by

$$
\begin{aligned}
& \alpha_{0}:=\varepsilon \\
& \alpha_{1}:=(f g)^{*} \\
& \alpha_{n+1}:=\left(f^{2^{n}} \alpha_{n} f g g^{2^{n}} \alpha_{n} f g\right)^{*} \quad(\text { for } n \in \mathbf{N} \backslash\{0\}) .
\end{aligned}
$$

Then for all $n \in \mathbf{N}$ it holds that

$$
\operatorname{sh}\left(L\left(\alpha_{n}\right)\right)=n .
$$

Proof. To identify the star height of a language given by a regular expression, one has to prove the nonexistence of equivalent expressions of lower star height. Here we are forced to give a proof for every parameter $n \in \mathbb{N}$. The technique applied in [4] (cf. also [14] for a similar approach) can be used to obtain this 
result. Here we only sketch the proof. For every $n \in \mathbb{N} \backslash\{0\}$, let $\mathcal{K}_{n}$ be a class of regular languages which satisfies the following three conditions (a), (b), and (c):

(a) For every language $L$ in $\mathcal{K}_{n}$

$$
\exists z \forall w \in L:|w|_{f}-|w|_{g}=z,
$$

where $|w|_{f}$ and $|w|_{g}$ denote the number of occurrences of $f$ and $g$, respectively, in the word $w$.

(b) For $m, n \in \mathbf{N} \backslash\{0\}$ let $w_{(n, m)} \in\{f, g\}^{*}$ be given by

$$
\begin{aligned}
& w_{(1, m)}:=f g \\
& w_{(n+1, m)}:=f^{2^{n}}\left(w_{(n, m)}\right)^{m} f g g^{2^{n}}\left(w_{(n, m)}\right)^{m} f g .
\end{aligned}
$$

For every $n \in \mathbb{N} \backslash\{0\}$, the $n$-subword index set of a language $L$ is

$$
\mathbf{T}_{n}^{L}:=\left\{m \in \mathbf{N} \backslash\{0\} \mid\left(w_{(n, m)}\right)^{m} \text { is a subword of a word in } L\right\} .
$$

The cardinality of this set, which is called $n$-subword index of $L$, must be infinite for each $L$ in $\mathcal{K}_{n}$ :

$$
\left|\mathbf{T}_{n}^{L}\right|=\infty
$$

i. e. there are (for every index $n$ of $\mathcal{K}_{n}$ ) infinitely many subwords of the form $\left(w_{(n, m)}\right)^{m}$ in $L$.

(c) Every element $L \in \mathcal{K}_{n}$ is minimal with respect to the star height among all languages which satisfy the conditions (a) and (b), i. e. for all regular languages $L^{\prime}$ over $\{f, g\}$ which also fulfil conditions (a) and (b) it holds that

$$
\operatorname{sh}(L) \leq \operatorname{sh}\left(L^{\prime}\right) .
$$

Thus all languages in $\mathcal{K}_{n}$ have the same star height.

It is easy to see that, for every $n \in \mathbf{N} \backslash\{0\}, L\left(\alpha_{n}\right)$ (cf. (1)) has properties (a) and (b). Hence, $\operatorname{sh}(L) \leq n$ for every $L \in \mathcal{K}_{n}$. The proof of the reverse inequation is shown by induction on $n$. For the case where $n=1$ this follows from the fact that every infinite regular language has a star height of at least 1 . For the inductive step we consider a decomposition of $L \in \mathcal{K}_{n+1}$ in a finite union of expressions of the form

$$
\gamma_{0}^{*} \gamma_{1} \gamma_{2}^{*} \gamma_{3} \ldots \gamma_{2 k-1} \gamma_{2 k}^{*}
$$

with $\operatorname{sh}\left(\gamma_{i}\right)<\operatorname{sh}(L)$ and verify that there is an index $i_{0}$ such that $\gamma_{i_{0}}$ meets (a) and (b) for the parameter $n$. With the inductive assumption we conclude $\operatorname{sh}(L) \geq n+1$. 


\section{Nested WHILE loops in Dijkstra schemes}

We now consider Dijkstra schemes with nested WHILE loops. We want to know whether it is possible to restrict the number of nested WHILE loops if we do not use coding mechanisms like in recursion theory, and if we do not require any special data structures.

We will show that such a limit does not exist in general. To this aim we exploit our characterization of Dijkstra scheme equivalence by formal languages and the star height property of regular languages. According to our preliminary definitions, the proof must be founded on a fixed finite signature of function and predicate symbols. Before studying this situation we consider the simpler case where the set of predicate symbols may become arbitrarily large. In this case it suffices to consider the value language $\operatorname{val}(S)$ of a Dijkstra scheme $S$ to establish the connection to formal language theory. It collects all execution paths of $S$, represented by the sequence of function symbols as they are applied, and is defined as the homomorphic image of the Dijkstra scheme language $L_{S}$ under the homomorphism which erases all Boolean vectors.

Proposition 9. (Value language of a Dijkstra scheme) Let $\Omega$ be a set of unary function symbols and $R \subseteq \Omega^{*}$ be an arbitrary non-empty regular language over $\Omega$. Then there exist

- a set $\Pi_{R}$ of predicate symbols

- and a Dijkstra scheme $S_{R} \in \operatorname{Dij}\left(\Omega, \Pi_{R}\right)$

such that $\operatorname{val}\left(S_{R}\right)=R$.

Proof. The proof is an easy induction on the set $\mathbf{R E}(\Omega)$ of all regular expressions over $\Omega$, where for the inductive step we assume that the sets of predicate symbols of the constituent schemes are disjoint and where we obtain one of the schemes

- if $p$ then $S_{R_{1}}$ else $S_{R_{2}}$ fi with a new predicate symbol $p$ for the case " $R_{1} \cup$ $R_{2}$ "

- $\left(S_{R_{1}} ; S_{R_{2}}\right)$ for " $R_{1} \cdot R_{2}$ "

- while $p$ do $S_{R_{1}}$ done with a new predicate symbol $p$ for the case " $R_{1}^{* \text { ". }}$

Note that the WHILE nesting depth of the resulting scheme coincides with the star height of the regular expression representing $R$.

Corollary 10. (Star height of Dijkstra scheme languages with infinite signatures) Let $\Omega$ be a set of function symbols with at least two elements and $\Pi$ be an arbitrary large set of predicate symbols. Then for every $n \in \mathbf{N}$ there exists a Dijkstra scheme $S_{n} \in \operatorname{Dij}(\Omega, \Pi)$ such that

$$
\operatorname{sh}\left(L_{S_{n}}\right)=n,
$$

i. e. the star height of Dijkstra scheme languages over infinite signatures is unbounded. 
Proof. We use the following result, cited in Section 4: In the class of regular languages over an alphabet with at least two elements there exists, for every number $n \in \mathbf{N}$, a regular language $L_{n}$ such that $\operatorname{sh}\left(L_{n}\right)=n$. Let $n \in \mathbf{N}$, and let $\alpha$ be a regular expression with $L(\alpha)=L_{n}$ and $\operatorname{sh}(\alpha)=n$. According to Proposition 9, there exists (a set $\Pi$ of predicate symbols and) a Dijkstra schema $S$ with $\operatorname{val}(S)=L_{n}$, constructed inductively on the structure of $\alpha$. Because its value language $\operatorname{val}(S)$ is a homomorphic image of the scheme language $L_{S}$, Lemma 7 yields

$$
\operatorname{sh}\left(L_{S}\right) \geq \operatorname{sh}(\operatorname{val}(S))=n .
$$

As mentioned above, since $S$ has been built up according to $\alpha$, it contains at most $n$ nested wHILE loops. On the other hand, only wHILE loops yield a contribution to the star height of the scheme language $L_{S}$. Thus we obtain

$$
\operatorname{sh}\left(L_{S}\right) \leq n
$$

and hence, by (2),

$$
\operatorname{sh}\left(L_{S}\right)=n .
$$

The question arises whether it is really necessary to introduce new predicate symbols, as in the proof of Theorem 9 . If it was possible to reuse them, then our proof could be based on a fixed signature. The following example illustrates the difficulties.

Example 11. Let $\Pi:=\{p\}$ and $\Omega:=\{f, g, h\}$. We consider the Dijkstra schemes $S_{1}$ and $S_{2}$ over this signature where

$$
S_{1}:=\text { if } p \text { then } f \text { else } g \text { fi }
$$

and

$$
S_{2}:=h \text {. }
$$

Then we get $L_{S_{1}}=\{1 f 0,1 f 1,0 g 0,0 g 1\}$ and $L_{S_{1}}=\{0 h 0,0 h 1,1 h 0,1 h 1\}$ and therefore $\operatorname{val}\left(S_{1}\right)=\{f, g\}$ and $\operatorname{val}\left(S_{2}\right)=\{h\}$.

If in the case " $R_{1} \cup R_{2}$ " of the above construction we would not introduce a new predicate symbol then we would obtain the Dijkstra scheme

$$
S=\text { if } p \text { then (if } p \text { then } f \text { else } g \text { fi) else } h \text { fi },
$$

which has the scheme language $L_{S}=\{1 f 0,1 f 1,0 h 0,0 h 1\}$ and thus the value language $\operatorname{val}(S)=\{f, h\}$. But then

$$
\operatorname{val}(S)=\{f, h\} \neq\{f, g, h\}=\operatorname{val}\left(S_{1}\right) \cup \operatorname{val}\left(S_{2}\right) .
$$

The reason for this is simply that $g$ becomes never applied in any interpretation because of the repeated use of the predicate symbol $p-S$ is not free. 
Now we present our proof of the hierarchy result with a fixed signature. We assume that we have at least one predicate symbol $p$ and at least two function symbols. In the discussion at the end of the paper we will explain why we cannot extend our proof technique to signatures where we have one function symbol only.

The set of predicate symbols which we need in the proof of Theorem 9 must contain at least as many symbols as the number of occurrences of + and ${ }^{*}$ in the regular expression where we start from. To restrict the number of predicate symbols we should sparingly use the symbol + , and we should reuse predicate symbols. The above example shows that such a reuse can at best be accomplished by employing free Dijkstra schemes, i. e. Dijkstra schemes where between two condition evaluations a computation (function application) must take place. This can be achieved by appending function symbols after WHILE loops.

An appropriate family $\left(S_{n}\right)_{n \in \mathbf{N}}$ of Dijkstra schemes over $\Omega=\{f, g\}$ and $\Pi:=\{p\}$ is given as follows. For every $n \in \mathbf{N}$, let

$$
f^{2^{n}}:=\underbrace{f ; \ldots ; f}_{2^{n} \text { times }}
$$

( $g^{2^{n}}$ analogously). Then $\left(S_{n}\right)_{n \in \mathrm{N}}$ is defined as

$$
\begin{aligned}
S_{0} \quad:= & \text { while }(p \wedge \neg p) \text { do } f ; g \text { done } \\
S_{1}:= & \text { while } p \text { do } f ; g \text { done; } \\
S_{n+1}:= & \text { while } p \text { do } \\
& \quad f^{2^{n}} ; S_{n} ; f ; g ; g^{2^{n}} ; S_{n} ; f ; g ; \\
& \text { done; }
\end{aligned}
$$

(where $n \geq 1$ ). Now the following theorem holds:

Theorem 12. (Star height of Dijkstra scheme languages over a fixed signature) Let $\Omega:=\{f, g\}$ and $\Pi:=\{p\}$. For $n \in \mathbf{N}$ let $S_{n} \in \operatorname{Dij}(\Omega, \Pi)$ be the Dijkstra schemes defined in (3). The Dijkstra scheme language $L_{S_{n}}$ has the following property:

$$
\operatorname{sh}\left(L_{S_{n}}\right)=n
$$

Proof. An easy induction over $n \in \mathbb{N}$ shows for the value language $\operatorname{val}\left(S_{n}\right)$ :

$$
\operatorname{val}\left(S_{n}\right)=L\left(\alpha_{n}\right)
$$

where $\alpha_{n}$ is the regular expression defined in Lemma 8. According to Proposition 8 we get $\operatorname{sh}\left(\operatorname{val}\left(S_{n}\right)\right)=n$ (for every $n \in \mathbb{N}$ ). As in the proof of Corollary 10 , Lemma 7 on the star height of homomorphic images and the observation that only WHILE loops can contribute to the star height of a Dijkstra scheme language yield

$$
n \stackrel{(8)}{=} \operatorname{sh}\left(\operatorname{val}\left(S_{n}\right)\right) \stackrel{(7)}{\leq} \operatorname{sh}\left(L_{S_{n}}\right) \leq n
$$

which implies that $\operatorname{sh}\left(L_{S_{n}}\right)=n$. 
From this theorem we can deduce a corollary which shows clearly the effect of the different notions of equivalence (program equivalence, scheme equivalence) and of the encodings by means of special data structures. While from the standpoint of recursion theory the number of nested loops can be bounded, such a limit does not exist from the standpoint of program scheme theory (because otherwise there would exist a limit on the star height of Dijkstra scheme languages). We express the main result of this section:

Corollary 13. (The WHILE Hierarchy of Dijkstra schemes) The hierarchy of nested WHIL loops in Dijkstra schemes is strict, i. e. for every $n \in \mathbf{N}$ there exists a Dijkstra scheme $S_{n+1}$ such that $S_{n+1}$ uses $n+1$ nested WHILE loops and $S_{n+1}$ cannot be equivalent to any Dijkstra scheme with less than $n+1$ nested WHILE loops.

\section{Conclusion and Discussion}

Conclusion: By combining two well-known techniques we characterized the equivalence of Dijkstra schemes which respect to the inductive structure of the class of Dijkstra schemes. We have shown, by considering the star height of Dijkstra scheme languages, that the renounce of coding mechanism and special data structures leads to an infinite hierarchy concerning the number of nested WHILE loops.

Discussion: Unfortunately Theorem 12 does not express anything about minimal signatures, i. e. signatures with one predicate symbol and one function symbol only. Since value languages of a Dijkstra scheme over such a signature are regular languages over a one-letter alphabet, the star height of the value language can only be 0 or 1 . So the technique we used in our proof can not be extended to such a signature, because the inequality

$$
\operatorname{sh}\left(\operatorname{val}\left(S_{n}\right)\right) \leq \operatorname{sh}\left(L_{S_{n}}\right)
$$

degenerates to $0 \leq \operatorname{sh}\left(L_{S_{n}}\right)$ or $1 \leq \operatorname{sh}\left(L_{S_{n}}\right)$, respectively. Thus the question is still open in this setting. Since it suffices to identify languages of arbitrary star height in the homomorphic images of the scheme languages, a possible approach might be a homomorphism which erases the function symbols instead of the Boolean vectors, yielding a regular language over the two-letter alphabet of truth values.

Acknowledgements: We would like to thank Klaus Indermark for the premise of this work, as well as Markus Mohnen and Thomas Wilke for the effort of reading a draft version of this paper.

\section{References}

1. Corrado Böhm and Giuseppe Jacopini. Flow diagrams, Turing machines and languages with only two formation rules. Communications of the ACM, 9(5):366-371, 1966. 
2. Steven Brown, David Gries, and Thomas Szymanski. Program schemes with pushdown stores. SIAM Journal on Computing, 1:242-268, 1972.

3. Rina S. Cohen and Janusz A. Brzozowski. General properties of star heigt of regular events. Journal of Computer and System Sciences, 4:260-280, 1970.

4. F. Dejean and M. P. Schützenberger. On a question of Eggan. Information and Control, 9:23-25, 1966.

5. L. C. Eggan. Transition graphs and the star-height of regular events. The Michigan Mathematical Journal, 10:385-397, 1963.

6. Iu. I. Ianov. On matrix program schemes. Communications of the $A C M, 12(1): 3-6$, 1958.

7. Iu. I. Ianov. On the equivalence and transformation of program schemes. Communications of the $A C M, 10(1): 8-12,1958$.

8. Iu. I. Ianov. The logical schemes of algorithms. Problems of Cybernetics, 1:82-140, 1960.

9. Klaus Indermark. On a class of schematic languages. In R. Aguilar, editor, Formal Languages and Programming, Proceedings of a Seminar Organized by UAM-IBM Scientific Center, pages 1-13, 1975.

10. S. C. Kleene. General recursive functions of natural numbers. Mathematische Annalen, 112:727-742, 1936.

11. D. C. Luckham, D. M. R. Park, and M. S. Paterson. On formalised computer programs. Journal of Computer and System Sciences, 4(3):220-249, 1970.

12. Michael S. Paterson and Carl E. Hewitt. Comparative schematology. Technical Report AI memo 201, MIT AI Lab, Publications Office, 545 Technology Sq. Cambridge, MA $02139,1970$.

13. Joseph D. Rutledge. On Ianov's program schemata. Journal of the $A C M, 11(1): 1-9$, 1964.

14. Arto Salomaa. Jewels of formal language theory. Computer Science Press, 1981. 